\title{
Confidence Building Among Correlated Streams in Multimedia Surveillance Systems
}

\author{
Pradeep K. Atrey ${ }^{1}$, Mohan S. Kankanhalli ${ }^{2}$, \\ and Abdulmotaleb El Saddik ${ }^{1}$ \\ ${ }^{1}$ School of Information Technology and Engineering, \\ University of Ottawa, Canada \\ $\{$ patrey, abed\}@mcrlab.uottawa.ca \\ ${ }^{2}$ Department of Computer Science, School of Computing, \\ National University of Singapore, Republic of Singapore \\ mohan@comp.nus.edu.sg
}

\begin{abstract}
Multimedia surveillance systems utilize multiple correlated media streams, each of which has a different confidence level in accomplishing various surveillance tasks. For example, the system designer may have a higher confidence in the video stream compared to the audio stream for detecting humans running events. The confidence level of streams is usually precomputed based on their past accuracy. This traditional approach is cumbersome especially when we add a new stream in the system without the knowledge of its past history. This paper proposes a novel method which dynamically computes the confidence level of new streams based on their agreement/disagreement with the already trusted streams. The preliminary experimental results show the utility of our method.
\end{abstract}

\section{Introduction}

Current surveillance systems often utilize multiple types of sensors like microphones [1, motion detectors [2] and RFIDs [3] etc in addition to the video cameras. As different sensors have different capabilities of performing various surveillance tasks, the designer of a multimedia surveillance system usually has different confidence levels in the evidences obtained based on the data of dissimilar sensors (we call sensor's data to be the "media streams" from now onwards) for accomplishing various tasks. For instance, the system designer may have higher confidence in a video stream compared to an audio stream for detecting faces, and may also have high confidence in an audio stream for detecting talking/shouting events.

In order to accomplish any surveillance task, the system assimilates relevant media streams. As the different streams have different confidence levels associated for accomplishing different tasks, it is important to utilize the confidence information of streams in their assimilation by appropriately assigning the weights to them [4. The confidence in a stream is related to its accuracy. The higher 
the accuracy of a stream, higher the confidence we would have in it. In the assimilation process, it makes sense to give more weight to a stream which has a higher confidence factor.

However, the computation of confidence information for each stream is cumbersome especially when we dynamically add the new streams to a multimedia surveillance system. The usual approach for determining the confidence in a stream is to first compute, in advance, its accuracy and then assign the confidence level to it based on its accuracy. This is often difficult because the system may provide different accuracies for different events when detected based on different media streams. Precomputation of accuracies of all the streams, that too for all events under different contexts, requires significant amount of training and testing, which is often tedious and time consuming. Moreover, for the streams which are added later in the system, there is no way to find their past accuracy. Therefore, it is important to devise a method to dynamically determine the confidence levels of streams without precomputing it.

In this paper, we propose a novel method for dynamically computing the confidences in a newly deployed stream based on the knowledge of the existing "trusted" stream(s) and the agreement coefficient among the newly deployed stream and the existing trusted streams. We call a stream to be "trusted" if its confidence level is greater than a threshold. The agreement coefficient between the streams is computed based on how agreeing or disagreeing the evidences obtained based on them have been in the past.

To illustrate our core idea, we provide the example of TV news channels. Let we follow a trusted CNN news channel. We also start watching an arbitrary XYZ news channel and compare the news content provided on both the channels. Over a period of time, our confidence in the XYZ channel will grow if the news content of both channels are found to be similar, and vice versa.

Rest of this paper is organized as follows. In section 2, we describe the related work. We formulate the problem of determining confidence in a stream in section 3. Section 4 presents our proposed method. We present the experimental results in section [5. Finally, section 6 concludes the paper with a discussion on the future work.

\section{Related Work}

In the past, the confidence has been used in the context of data management in sensor networks. Tatbul et al. [5] compute the confidence in a stream based on how it has helped in making the accurate decisions in the past. Tavakoli et al. [6] proposed a method for event detection that uses historical and spatial information in clusters in order to determine a confidence level that warrants a detection report with high confidence. Ioannou et al. 7] also employed a confidence-based fusion strategy to combine multiple feature cues for facial expression recognition. However, the works at [5], 6] and [7] did not elaborate on how the confidence value is used in the integration of information. 
Siegel and $\mathrm{Wu}[8]$ has pointed out the importance of considering the confidence in sensor fusion. The authors have used the Dempster-Shafer (D-S) theory of evidence to fuse the confidences. In contrast, we propose a model for confidence fusion by using a Bayesian formulation because it is both simple and computationally efficient 4 .

In all the past works, the confidence in streams has been computed based on their past accuracy. This work is different from the past works in that, our method computes the confidence level of streams based their agreement/ disagreement with the trusted streams. Agreement coefficient among streams is computed based on how concurring or contradictory evidences they provide. Agreement coefficient between any two streams is different from mutual information [9] between them in that the former connotes the measure of mutual consistency or contradiction between the two streams while the latter implies how much information does one stream convey about another one.

\section{Problem Formulation}

We formulate below the problem of determining the confidence level of a media stream:

M1. Sis a multimedia surveillance system designed for detecting a set $E$ of events, and it consists of $n \geq 1$ heterogeneous sensors that capture data from the environment. Let $\mathbf{M}^{n}=\left\{M_{1}, M_{2}, \ldots, M_{n}\right\}$ be the media streams obtained from $n$ sensors.

M2. For $1 \leq i \leq n$, let $0<p_{i}(t)<1$ be the probability of occurrence of an event based on individual $i^{t h}$ media stream at time instant $t$. The $p_{i}(t)$ is determined by first extracting the features from media stream $i$ and then by employing an event detector (e.g. a trained classifier) on them. Also, let $P_{\Phi}(t)$ be the 'fused probability' of occurrence of the event at time $t$ based on a subset $\Phi \in \mathcal{P}\left(\mathbf{M}^{n}\right)$ of media streams. The 'fused probability' is the overall probability of occurrence of the event based on a group of media streams [4.

M3. For $1 \leq i \leq n$, let $0<f_{i}(t)<1$ be the system designer's confidence in the $i^{\text {th }}$ stream at time instant $t$. The confidence in at least one media stream is learned by experimentally determining its accuracy. More the accurate results we obtain based on a stream, more the confidence we would have in it. Also, there exists a subset $T \subseteq \mathbf{M}^{n}$ (with $|T| \geq 1$ ) of streams in which the confidence level of streams is greater than or equal to a threshold (say $\left.F_{\text {spec }}\right)$. We call them to be the "trusted" media streams.

We make the following assumptions:

$\mathcal{A} 1$. All sensing devices capture the same environment (but optionally, the different aspects of the environment) and provide correlated observations.

$\mathcal{A} 2$. The system designer's confidence level in each of the media streams is at least 0.5. This assumption is reasonable since it is not useful to employ a media device which is found to be inaccurate more than half of the time. 
$\mathcal{A} 3$. The fused probability of the occurrence of event and the overall confidence increase monotonically as the more concurring evidences are obtained based on the streams.

The objective is to determine the confidence level $f_{i}(t+1)$ of a new non-trusted stream $M_{i}$ at time instant $t+1$ given that its confidence level at time instant $t$ is $f_{i}(t)$. In absence of any prior information, $f_{i}(0)=\epsilon$ (a positive infinitesimal).

\section{Proposed Method}

The proposed method determines the confidence in a new non-trusted stream using its "Agreement Coefficient" with the trusted stream(s). The agreement coefficient between the two streams is computed based on whether the evidence obtained by the system using them are concurring or contradictory.

\subsection{Modelling of the Agreement Coefficient}

Let the measure of agreement among the media streams at time $t$ be represented by a set $\Gamma(t)$ which is expressed as:

$$
\Gamma(t)=\left\{\gamma_{i k}(t)\right\}
$$

where, the term $-1 \leq \gamma_{i k}(t) \leq 1$ is the agreement coefficient between the media streams $M_{i}$ and $M_{k}$ at time instant $t$.

The system computes the agreement coefficient $\gamma_{i k}(t)$ between the media streams $M_{i}$ and $M_{k}$ at time instant $t$ by iteratively averaging the past agreement coefficients with the current observation. Precisely, $\gamma_{i k}(t)$ is computed as:

$$
\gamma_{i k}(t)=\frac{1}{2}\left[\left(1-2 \times a b s\left(p_{i}(t)-p_{k}(t)\right)\right)+\gamma_{i k}(t-1)\right]
$$

where, $p_{i}(t)=P\left(E_{t} \mid M_{i}\right)$ and $p_{k}(t)=P\left(E_{t} \mid M_{k}\right)$ are the individual probabilities of occurrence of event $E$ based on media streams $M_{i}$ and $M_{k}$, respectively, at time $t \geq 1$; and $\gamma_{i j}(0)=1-2 \times a b s\left(p_{i}(0)-p_{k}(0)\right)$. These probabilities represent decisions about the events. Exactly same probabilities would imply full agreement $\left(\gamma_{i k}=1\right)$ whereas totally dissimilar probabilities would mean that the two streams fully contradict each other $\left(\gamma_{i k}=-1\right) 4$.

The agreement coefficient between two sources $\mathbf{M}^{i-1}$ and $M_{i}$ is modelled as:

$$
\gamma_{M_{i}, \mathbf{M}^{i-1}}=\frac{1}{i-1} \sum_{s=1}^{i-1} \gamma_{s i}
$$

where, $\gamma_{s i}$ for $1 \leq s \leq i-1,1<i \leq n$ is the agreement coefficients between the $s^{t h}$ and $i^{t h}$ media streams. The agreement fusion model given in equation (3) is based on average-link clustering. In average-link clustering, we consider the distance between one cluster and another cluster to be equal to the average distance from any member of one cluster to any member of the other cluster [10]. In our case, a group $\mathbf{M}^{i-1}$ of $i-1$ media streams is one cluster and we find the average distance of new $i^{\text {th }}$ media stream with this cluster. 


\subsection{Confidence Fusion}

The confidence fusion refers to the process of finding the overall confidence in a group of media streams where the individual media streams have their own confidence level. Given that the two streams $M_{i}$ and $M_{k}$ have their confidence levels $f_{i}$ and $f_{k}$, respectively, the system uses a Bayesian method to fuse the confidence levels in individual streams. The overall confidence $f_{i k}$ in a group of two media streams $M_{i}$ and $M_{k}$ is computed as follows:

$$
f_{i k}=\frac{f_{i} \times f_{k}}{f_{i} \times f_{k}+\left(1-f_{i}\right) \times\left(1-f_{k}\right)}
$$

In the above formulation, we make two assumptions. First, we assume that the system designer's confidence level in each of the media streams is more than 0.5 (Refer to assumption $\mathcal{A} 2$, in section 3). Second, although the media streams are correlated in their decisions; we assume that they are mutually independent in terms of their confidence levels 4 .

For $n$ number of media streams, the overall confidence is iteratively computed. Let $F_{i-1}$ be the overall confidence in a group of $i-1$ streams. By fusing the confidence $f_{i}$ of $i^{\text {th }}$ stream with $F_{i-1}$, the overall confidence $F_{i}$ in a group of $i$ streams is computed as:

$$
F_{i}=\frac{F_{i-1} \times f_{i}}{F_{i-1} \times f_{i}+\left(1-F_{i-1}\right) \times\left(1-f_{i}\right)}
$$

\subsection{Confidence Building Method}

Given a set $T$ of trusted media streams, the confidence level of the media stream $M_{i}$ is computed as follows:

- Using a voting strategy, we divide the set $T$ of trusted media streams into two subsets $T_{1}$ and $T_{2}$ (as shown in figure1). This division is performed based on whether, at the current instant, the evidence obtained by the system using these two subsets are concurring or contradictory. Precisely, the subset, based on which, the system concludes in favor of the occurrence of event $E$ with more than 0.50 probability are put in set $T_{1}$ and the rest in set $T_{2}$.

- The agreement coefficients $\gamma_{i, T_{1}}$ (between the stream $M_{i}$ and the subsets $T_{1}$ ) and $\gamma_{i, T_{2}}$ (between the stream $M_{i}$ and the subset $T_{2}$ ) are computed as described in section 4.1 (equation 3).

- Next, the system computes the overall confidence $F_{T_{1}}$ and $F_{T_{2}}$ in the subsets $T_{1}$ and $T_{2}$, respectively, (using equation 5).

- Finally, the system computes the confidence $f_{i}(t+1)$ in the $i^{\text {th }}$ stream at time instant $t+1$ as follows:

$$
f_{i}(t+1)= \begin{cases}F_{T_{1}} \times \frac{f_{i}(t) \cdot e^{\alpha, \gamma_{i}, T_{1}}(t)}{f_{i}(t) \cdot e^{\alpha \cdot \gamma_{i}, T_{1}(t)}+\left(1-f_{i}(t)\right) \cdot e^{-\alpha \cdot \gamma_{i}, T_{1}}(t)} & \text { if } F_{T_{1}} \times \gamma_{i, T_{1}}(t) \geq \\ F_{T_{2}} \times \frac{f_{i}(t) \cdot e^{\alpha, \gamma_{i}, T_{2}(t)}}{f_{i}(t) \cdot e^{\alpha \cdot \gamma_{i}, T_{2}(t)}+\left(1-f_{i}(t)\right) \cdot e^{-\alpha \cdot \gamma_{i}, T_{2}(t)}} & \text { otherwise }\end{cases}
$$




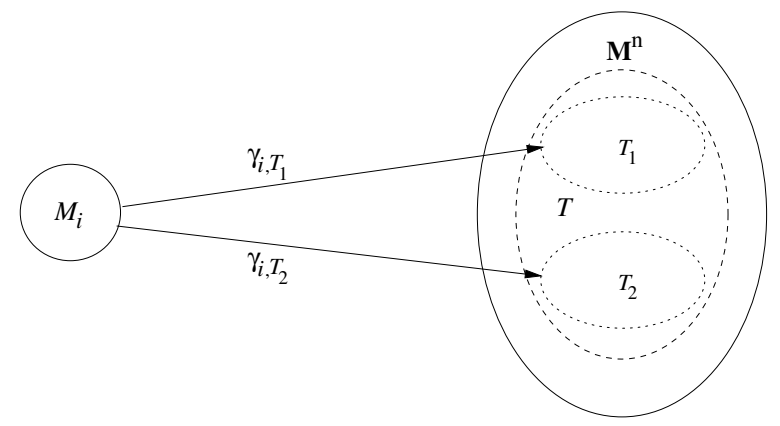

Fig. 1. The agreement coefficient between stream $M_{i}$ and the subsets $T_{1}$ and $T_{2}$

The exponential terms $e^{\alpha \cdot \gamma_{i, T_{1}}(t)}$ and $e^{\alpha \cdot \gamma_{i, T_{2}}(t)}$ represent the growth in the confidence level at time $t . \alpha \in[0, \infty]$ is used as a growth rate with respect to overall confidence levels $F_{T_{1}}$ and $F_{T_{2}}$ of groups $T_{1}$ and $T_{2}$, respectively. The terms $\gamma_{i, T_{1}}(t)$ and $\gamma_{i, T_{2}}(t)$ denote the agreement coefficient at time $t$ between the $i^{t h}$ stream and the groups $T_{1}$ and $T_{2}$, respectively. In the above formulation, the denominator term acts as normalization factor to limit the confidence value within $[0,1]$. Note that if either $T_{1}$ or $T_{2}$ is found empty, their fused confidence levels ( $F_{T_{1}}$ and $F_{T_{2}}$, respectively) are considered to be of zero value.

\section{$5 \quad$ Experimental Results}

We show the utility of our method in a surveillance scenario. The surveillance environment is the corridor of our school building with a system goal to detect events such as humans running, walking and standing in the corridor. We use two video sensors (Canon VC-C50i cameras denoted by $V_{1}$ and $V_{2}$ ) to record the video from the two opposite ends of corridor as shown in figure 2, The two cameras are connected to a central PC (Pentium-IV 3.6 GHz). A Picolo-Pro video capture card is used to capture the image data.

For our experiments, we have used data of more than twelve hours which has been recorded using the system consisting of two video cameras. Over the period of more than twelve hours, the noticeable events occurred over for a period

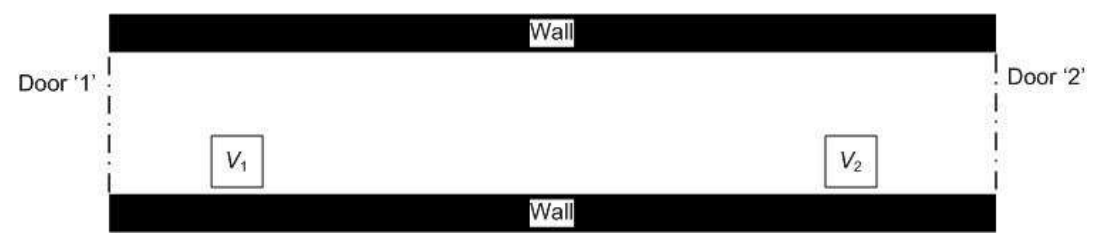

Fig. 2. The layout of the corridor under surveillance 
of 1079 seconds. The details of various events and their total durations are as follows - humans standing events for 139 seconds, walking events for 798 seconds and running events for 142 seconds.

The system detects these events by processing the video frames. The video processing involves background modeling and blob detection. The background modeling is performed using an adaptive Gaussian method 11. For blob detection, the system first segments the foreground from the background using simple 'matching' on the three RGB color channels, and then uses the morphological operations (erode and dilation) to obtain connected components (i.e. blobs). The matching is defined as a pixel value being within 2.5 standard deviations of the distribution. We assume that the blob of an area greater than a threshold corresponds to a human. An example of blob detection (with its bounding rectangle) in a humans "running" event is shown in figure 3. Once the bounding rectangle for each blob is computed, the middle point of the bottom edge of the bounding rectangle is mapped to the actual ground location using the caliberation information of the video cameras. This provides the exact ground location of human in the corridor at a particular time instant.

The system identifies the start and end of an event in video streams as follows. If a person moves towards the camera, the start of event is marked when the blob's area becomes greater than a threshold and the event ends when the blob intersects the image plane. However, if the person walks away from the camera, the start and end of the event is inverted. Based on the average distance travelled by human on the ground, a Bayes classifier is first trained and then used to classify an atomic-event to be one of the classes - standing, walking and running.

We present our preliminary results as follows. First, the system performed event detection and classification using only one stream i.e. video stream $V_{1}$. By comparing with the ground truth, we found overall accuracy of the video stream $V_{1}$ to be $68 \%$. Based on the accuracy, we assigned a confidence level 0.68 to the stream $V_{1}$, and designated it to be the "trusted" stream. Note that, in our experiments, the threshold value used for trusted stream is 0.65 . Determing the ideal value of this threshold is an issue which we will examine in the future

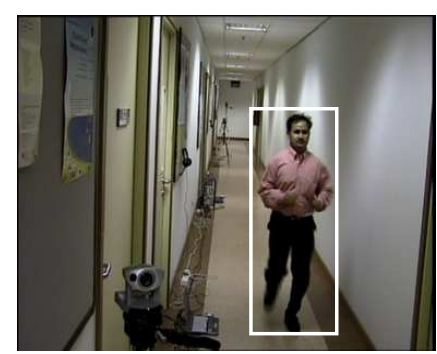

(a)

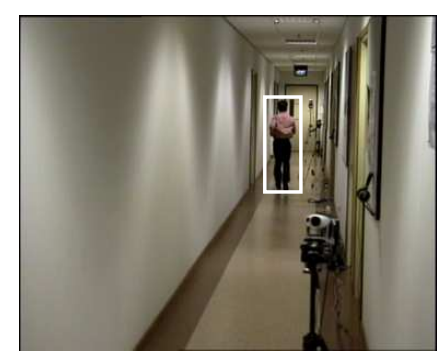

(b)

Fig. 3. An example: Bounding rectangles along the detected blobs in the video frames of (a) Camera 1 and (b) Camera 2, corresponding to a hummas "running" event 
work. Based on the agreement coefficient between the trusted stream $V_{1}$ and the other video stream $V_{2}$, the system uses our proposed method to compute the confidence in stream $V_{2}$.
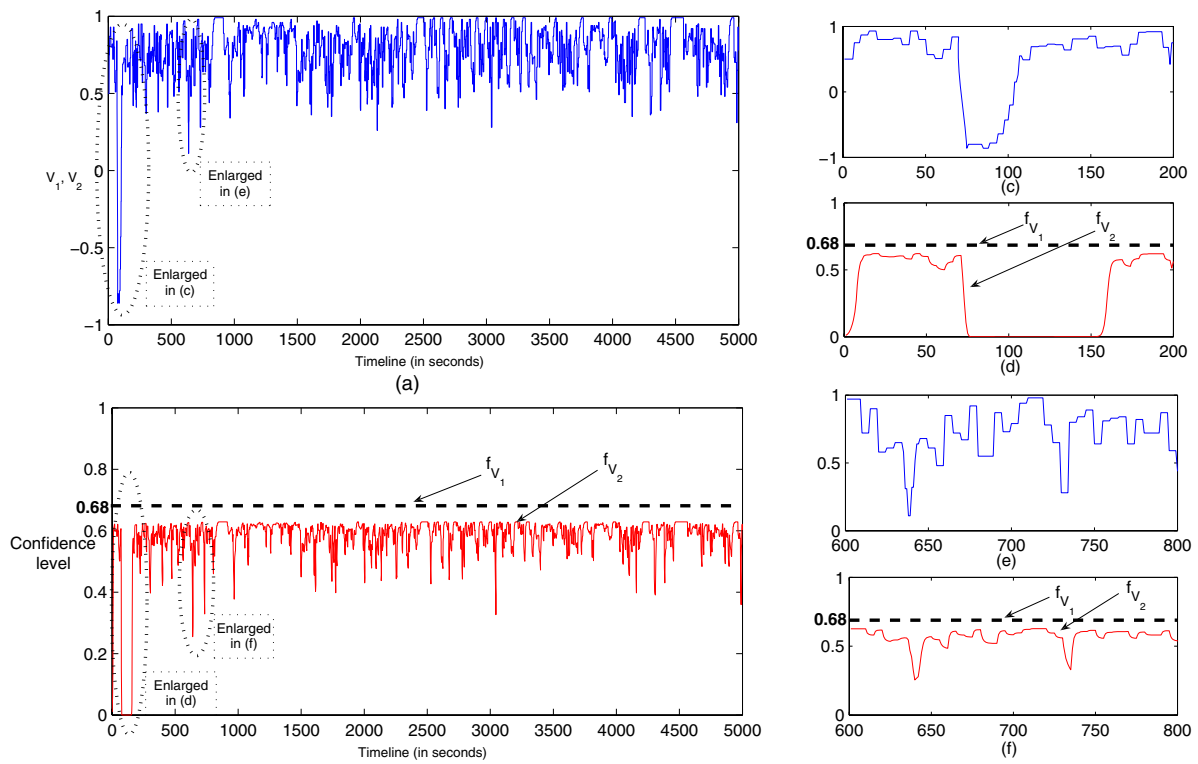

(b)

Fig. 4. Confidence building in $V_{2}$ stream

Timeline-based confidence building in the stream $V_{2}$ is shown in figure 4 . Figure 4(a) shows how the agreement coefficient between $V_{1}$ and $V_{2}$ varies along the timeline, and figure 4 (b) depicts how the confidence $f_{V_{2}}$ in $V_{2}$ evolves along the timeline. Figures 4(c)-4(f) show the enlarged portions of some parts of figures 4(a)-4(b). For example, figure 4(c) shows how the agreement coefficient $\gamma_{V_{1}, V_{2}}$ drops down below zero and then consequently figure 4(d) depicts that how confidence also decreases as the agreement coefficient decreases. Once the confidence level drops down at around $75^{t h}$ second along the timeline, the confidence $f_{V_{2}}$ in stream $V_{2}$ also drops to almost zero and it takes approximately another 90 seconds to regain the same confidence level. Similarly, as can be seen in figures 4(e)-4(f), the confidence level $f_{V_{2}}$ in stream $V_{2}$ decreases to 0.25 at time instant 642 and to 0.30 at time instant 740 as the agreement coefficient goes below 0.5 , however, in these two cases, the confidence level picks up early close to the confidence level of trusted stream compared to when the agreement coefficient becomes negative.

Note that we have set the value of the growth rate $\alpha$ to be 1 . With $\alpha=1$, the system could gain the confidence level in $V_{2}$ upto 0.63 . However, with higher growth rate $(\alpha \approx 5)$, this maximum achieved confidence can go up to the level of 
confidence in the trusted stream. Again, determining the ideal value of growth factor $\alpha$ is an issue which is out of scope of this paper and will be investigated in the future.

To verify the utility of our method, we compared the average confidence level of stream $V_{2}$ determined using our method with the confidence level which is computed based on its past accuracy. It is observed that both are comparable (0.58 vs 0.60$)$ as shown in Table 1

Table 1. Comparison of the proposed method with the traditional approach

\begin{tabular}{|l|l|}
\hline Method of computing confidence & Confidence level of $V_{2}$ \\
\hline Pre-computed confidence & 0.60 \\
Our method & 0.58 (Average value) \\
\hline
\end{tabular}

\section{Conclusions}

This paper proposes a novel method to dynamically compute the confidence levels of new media streams in a multimedia surveillance system. The confidence in a new stream is computed based on the fact whether it provides evidence which concurs or contradicts with the already trusted streams. Though the preliminary results have shown that the confidence level computed using our method is comparable with the confidence level determined based on the traditional approach (past accuracy), we need to investigate in detail how the dynamically varying confidence level can contribute towards more accurate overall results for event detection in multimedia surveillance systems. It will also be interesting to examine the utility of the proposed method for dissimilar sensors such as microphones and motion detectors, and also for the different kinds of events such as audio events - talking, shouting, door knocking and footsteps.

\section{Acknowledgements}

We thank Prof Ramesh Jain for suggesting this research problem.

\section{References}

1. Atrey, P.K., Maddage, N.C., Kankanhalli, M.S.: Audio based event detection for multimedia surveillance. In: IEEE International Conference on Acoustics, Speech, and Signal Processing. (2006) V813-816

2. Rama, K.G.S., Atrey, P.K., Singh, V.K., Ramakrishnan, K., Kankanhalli, M.S.: A design methodology for selection and placement of sensors in multimedia surveillance systems. In: The 4th ACM International Workshop on Video Surveillance and Sensor Networks, Santa Barbara, CA, USA (2006)

3. Prati, A., Vezzani, R., Benini, L., Farella, E., Zappi, P.: An integrated multi-modal sensor network for video surveillance. In: The ACM International Workshop on Video Surveillance and Sensor Networks, Singapore (2005) 95-102 
4. Atrey, P.K., Kankanhalli, M.S., Jain, R.: A framework for information assimilation in multimedia surveillance systems. ACM Multimedia Systems Journal (2006)

5. Tatbul, N., Buller, M., Hoyt, R., Mullen, S., Zdonik, S.: Confidence-based data management for personal area sensor networks. In: The Workshop on Data Management for Sensor Networks. (2004) 24-31

6. Tavakoli, A., Zhang, J., Son, S.H.: Group-based event detection in undersea sensor networks. In: Second International Workshop on Networked Sensing Systems, San Diego, California, USA (2005)

7. Ioannou, S., Wallace, M., Karpouzis, K., Raouzaiou, A., Kollias, S.: Confidencebased fusion of multiple feature cues for facial expresssion recognition. In: The 14th IEEE International Conference on Fuzzy Systems, Reno, Nevada, USA (2005) 207212

8. Siegel, M., Wu, H.: Confidence fusion. In: IEEE International Workshop on Robot Sensing. (2004) 96-99

9. Conaire, C.O., Connor, N.O., Cooke, E., Smeaton, A.: Detection thresholding using mutual information. In: International Conference on Computer Vision Theory and Applications, Setubal, Portugal (2006)

10. Jain, A.K., Murty, M.N., Flynn, P.J.: Data clustering: A review. ACM Computing Surveys 31(3) (1999) 264-323

11. Stauffer, C., Grimson, W.E.L.: Adaptive background mixture models for real-time tracking. In: IEEE Computer Society Conference on Computer Vision and Pattern Recognition. Volume 2., Ft. Collins, CO, USA (1999) 252-258 